\title{
Diencephalic syndrome: the ghost behind unexplained weight loss in children \\ N:YER
}

\author{
S. Trapani, A. Brambilla, B. Bortone \\ University of Florence - Anna Meyer Children Hospital, Florence (Italy)
}

\section{Background and aims}

Diencephalic syndrome is a rare cause of infant cachexia associated with suprasellar and third ventricular tumours. Severe emaciation is characteristic, whereas neurologic manifestations could appear later. Final prognosis is generally poor. We report two cases of diencephalic syndrome leading to different outcomes.

\section{Methods and Results}

GB, a 14-months girl and a AT, a 16-months boy, were admitted for severe failure to thrive, starting few months after weaning (Figure 1-2). Clinical examination documented severe hemaciation despite normal caloric intake; length and head circumference resulted appropriate. No abnormalities in cardiac, thoracic and abdominal examination were recorded. No superficial lymphadenopathies were detected. Laboratory work up confirmed normal baseline investigation, excluding signs of infections and common causes of intestinal malabsorption. Stool culture excluded bacterial, mycotic and parasitic infections. Peripheral blood smear evidenced normal count blood cell without achantocytes. Sweat test and autoimmunity markers gave negative result. Complete hormonal assessment was performed, revealing normal value of cortisol, ACTH, $\mathrm{TSH}, \mathrm{fT} 4$, IgF1, LH, FSH, $17 \mathrm{~B}$ oestradiol, testosterone and prolactin. We proceeded with Chest X-Ray and abdomen echography, both normal; hand X-ray confirmed appropriate skeletal age.

Neurologic examination revealed occasional hyperactivity in GB, hyperalertness and mild horizontal nystagmus in AT.

Brain-Magnetic Resonance (MR) was finally performed, documenting suprasellar large brain tumour showing positive enhancement after contrast infusion. The mass involved hypothalamic-pituitary region, extended to third ventricle; secondary lateral ventricle dilation was reported (Patient 1: Images A-B; Patient 2: Image C]. Histologic examination confirmed the diagnosis of low-grade astrocytomas. Both patients underwent chemotherapy, radiotherapy and debulking surgery. GB developed severe hydrocephalus and finally died 12 months after. AT showed a mild catch up growth after 4 months of chemotherapy, with a stable lesion and is still on therapy.

\section{Conclusions}

Diencephalic syndrome is a rare cause of infant cachexia with unfavourable outcome. In this clinical setting, diagnostic delay and misdiagnosis are common. Diagnostic pathway should deepen dietary anamnesis, highlighting progressive weight loss despite adequate caloric intake. Moreover, the presence of severe weight loss associated with preserved length and normal/increased cranial circumference is another element of high suspicion. Neurologic symptoms are usually not precocious; therefore, the absence of these signs should not prevent second-line investigation (brain MRI).
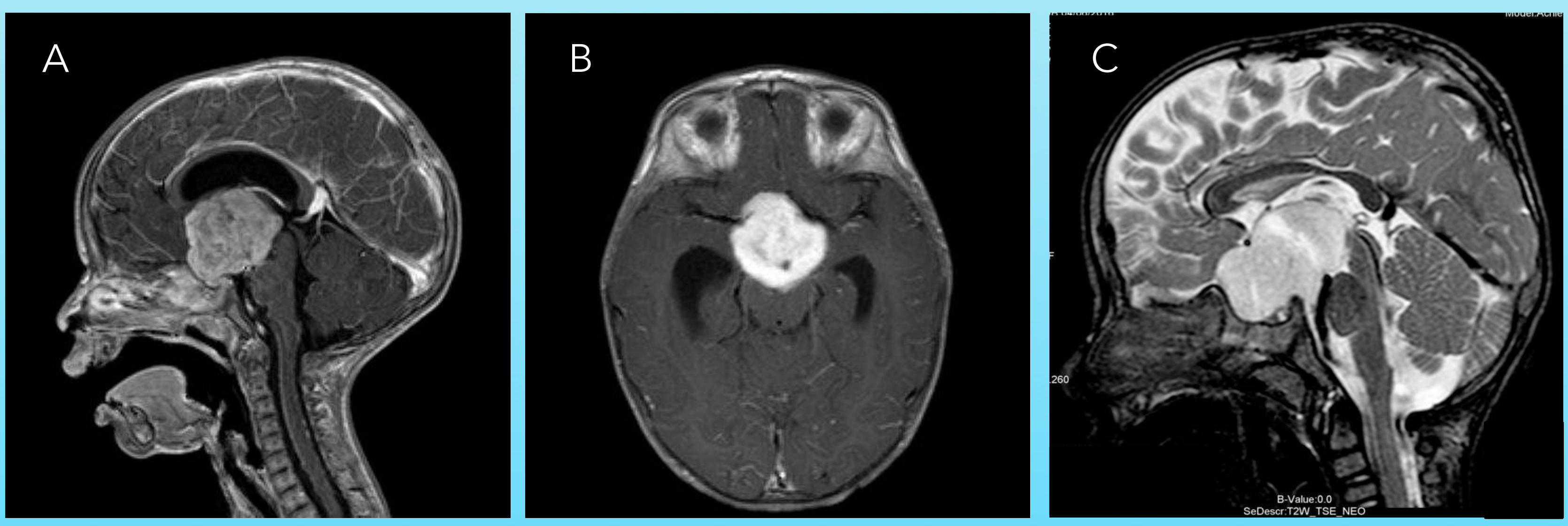

Copyright @ 2017 S. Trapani, University of Florence, Florence (Italy). E-mail: sandra.trapani@unifi.it

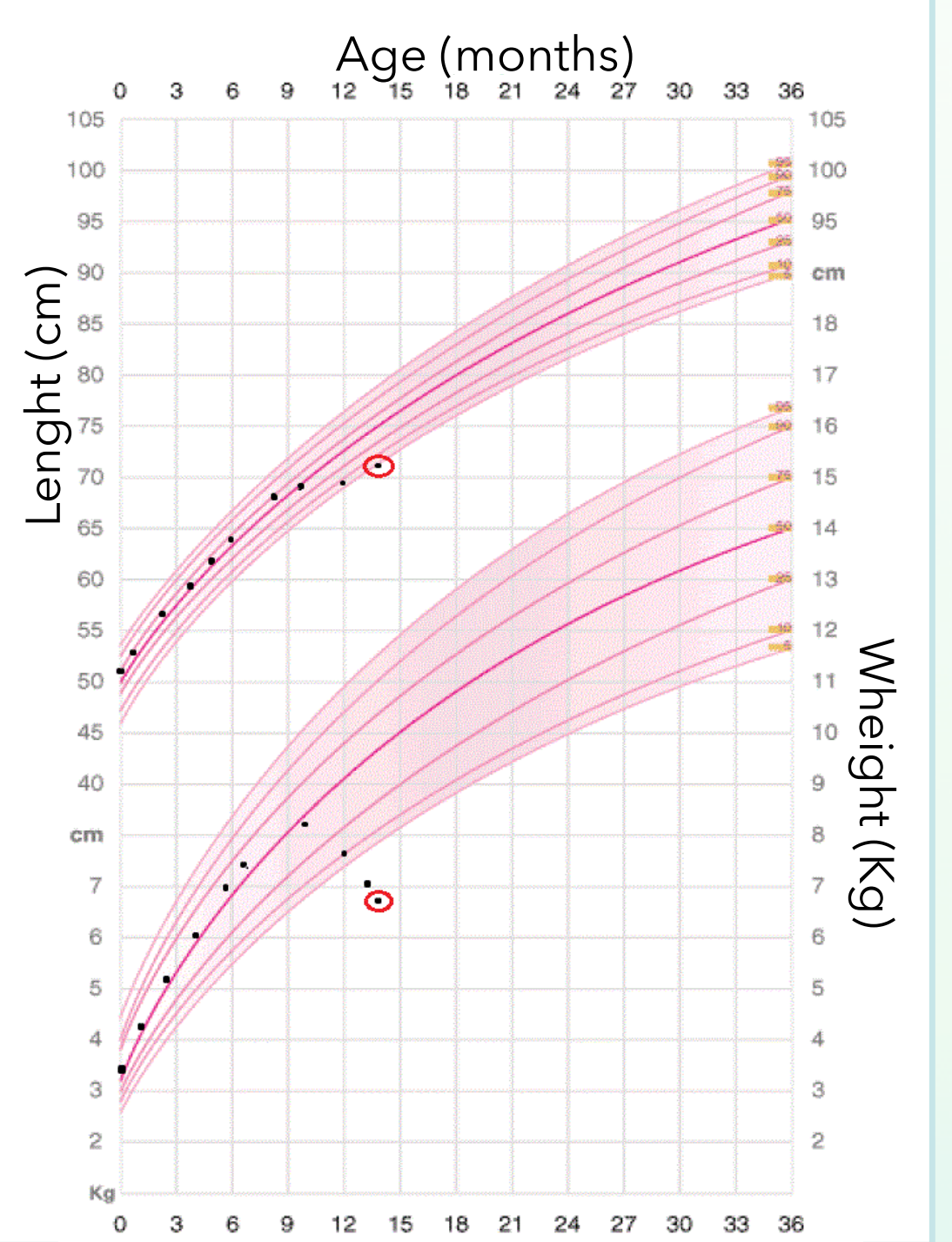

Figure 1 - Growth chart of Patient 1

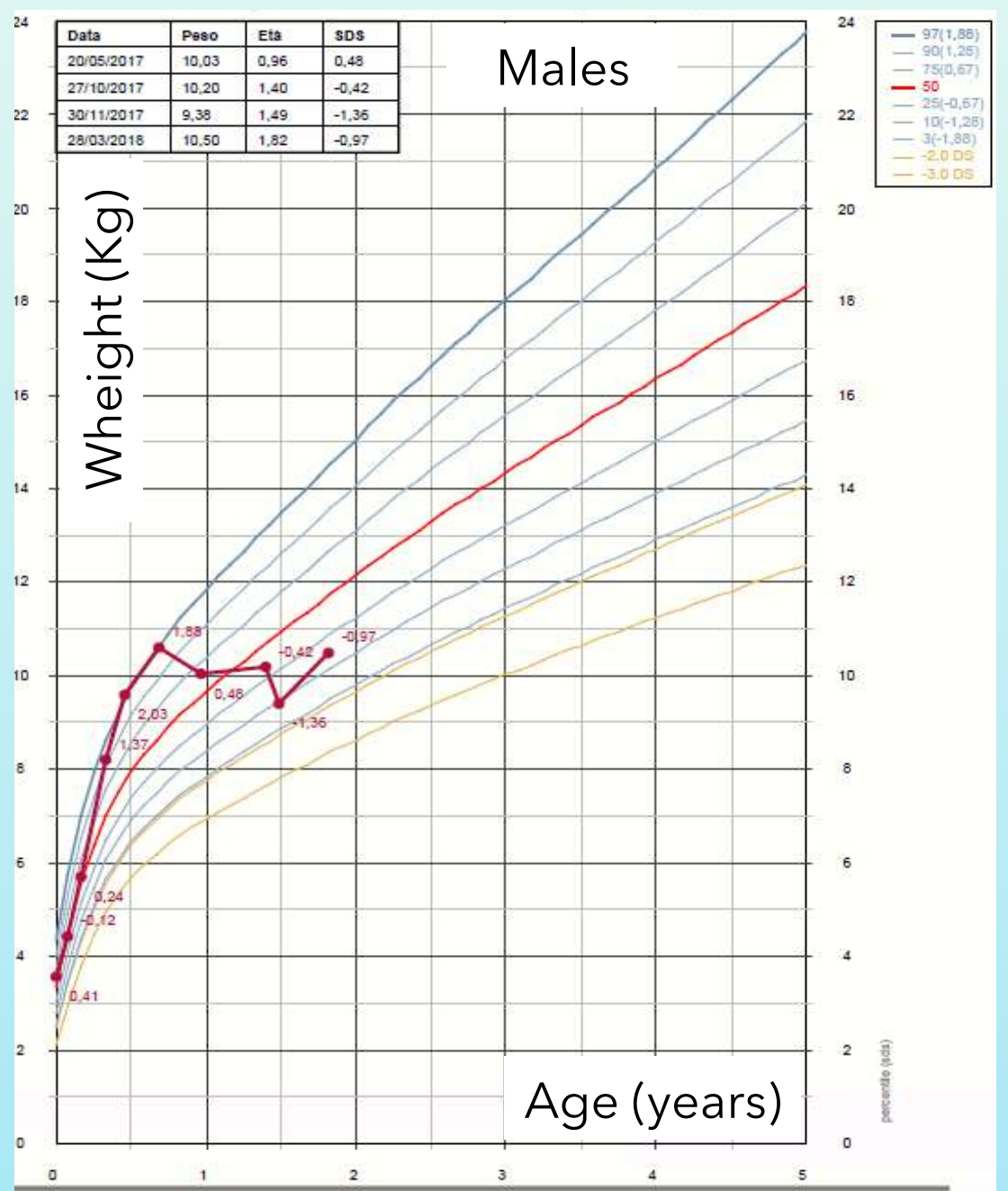

Figure 2 - Growth chart of Patient 2 